\title{
Multifocal demyelinating motor neuropathy and hamartoma syndrome associated with a de novo PTEN mutation
}

Boglarka Bansagi, MD, PhD, * Vietxuan Phan, PhD, * Mark R. Baker, PhD, FRCP, * Julia O’Sullivan, MSc, Matthew J. Jennings, MSc, Roger G. Whittaker, PhD, FRCP, Juliane S. Müller, PhD, Jennifer Duff, PhD, Helen Griffin, PhD, James A.L. Miller, PhD, MRCP, Grainne S. Gorman, MD, PhD, Hanns Lochmüller, MD, Patrick F. Chinnery, PhD, FMedSci, Andreas Roos, PhD, * Laura E. Swan, PhD,* and Rita Horvath, MD, PhD* Neurology ${ }^{\circledR}$ 2018;90:e1842-e1848. doi:10.1212/WNL.0000000000005566

\section{Abstract \\ Objective}

To describe a patient with a multifocal demyelinating motor neuropathy with onset in childhood and a mutation in phosphatase and tensin homolog (PTEN), a tumor suppressor gene associated with inherited tumor susceptibility conditions, macrocephaly, autism, ataxia, tremor, and epilepsy. Functional implications of this protein have been investigated in Parkinson and Alzheimer diseases.

\section{Methods}

We performed whole-exome sequencing in the patient's genomic DNA validated by Sanger sequencing. Immunoblotting, in vitro enzymatic assay, and label-free shotgun proteomic profiling were performed in the patient's fibroblasts.

\section{Results}

The predominant clinical presentation of the patient was a childhood onset, asymmetric progressive multifocal motor neuropathy. In addition, he presented with macrocephaly, autism spectrum disorder, and skin hamartomas, considered as clinical criteria for PTEN-related hamartoma tumor syndrome. Extensive tumor screening did not detect any malignancies. We detected a novel de novo heterozygous c.269T >C, p.(Phe90Ser) PTEN variant, which was absent in both parents. The pathogenicity of the variant is supported by altered expression of several PTEN-associated proteins involved in tumorigenesis. Moreover, fibroblasts showed a defect in catalytic activity of PTEN against the secondary substrate, phosphatidylinositol 3,4-trisphosphate. In support of our findings, focal hypermyelination leading to peripheral neuropathy has been reported in PTEN-deficient mice.

\section{Conclusion}

We describe a novel phenotype, PTEN-associated multifocal demyelinating motor neuropathy with a skin hamartoma syndrome. A similar mechanism may potentially underlie other forms of Charcot-Marie-Tooth disease with involvement of the phosphatidylinositol pathway.

\author{
Correspondence \\ Dr. Horvath \\ Rita.Horvath@ncl.ac.uk
}




\section{Glossary}

ASD $=$ autism spectrum disorder; CMT $=$ Charcot-Marie-Tooth disease; HNPP $=$ hereditary neuropathy with liability to pressure palsies; PHTS = PTEN hamartoma tumor syndrome; PI(3)K = phosphoinositide 3-kinase; $\mathbf{P I}(\mathbf{3}, \mathbf{4}) \mathbf{P}_{\mathbf{2}}=$ phosphatidylinositol 3,4,-trisphosphate; PIP $_{3}=$ phosphatidylinositol 3,4,5-trisphosphate; $\mathbf{P T E N}=$ phosphatase and tensin homolog located on chromosome 10.

Inherited peripheral neuropathies (Charcot-Marie-Tooth disease $[\mathrm{CMT}]$ ) encompass genetically heterogeneous sensory or motor nerve conditions. ${ }^{1}$ Next generation sequencing ${ }^{2}$ and proteomics ${ }^{3}$ are powerful and robust methodologic approaches to elucidate the underlying molecular cause and pathologic mechanisms.

Phosphatase and tensin homolog located on chromosome 10 (PTEN) is a tumor suppressor gene in which mutations are typically found in sporadic cancers and inherited tumor susceptibility conditions, such as the PTEN hamartoma tumor syndrome (PHTS). Macrocephaly, autism spectrum disorder (ASD), ataxia, tremor, and epilepsy have been reported in patients with PTEN mutations, while functional implications of this protein have been investigated in Parkinson and Alzheimer diseases. ${ }^{4,5}$ Various PTEN functions in neurons have been linked to its dynamic spatiotemporal distribution. Regulation of neuronal cell size, axonal outgrowth, synaptogenesis, and neuromuscular assembly are PTEN-controlled processes during neuronal development and regeneration after injury. Furthermore, in PTEN-deficient mice abnormal regulation of myelination has been observed in peripheral nerves. ${ }^{6,7}$

Here we describe a patient manifesting with multifocal demyelinating motor neuropathy and hamartoma syndrome in association with a de novo heterozygous PTEN mutation.

\section{Methods}

A 29-year-old patient had been born to nonconsanguineous Caucasian parents. Delayed motor development and speech were reported with facial asymmetry at 7 years of age. He developed focal weakness involving his right eye movements, his face, and contralateral extremities in the following years. By age 20, multiple livid papules and keloid scars appeared on his chest and he had bowel problems and learning difficulties.

Examination at age 29 revealed a large scaphocephalic head (macrocephaly), asymmetric face, and crowded teeth. The patient had upper thoracic scoliosis. Right oculomotor palsy, complete right-sided and partial left-sided facial palsy, and leftsided tongue atrophy were noted. He had nasal speech. There was both proximal and distal flaccid paresis in the left upper limb, with atrophy and areflexia. In the right arm, he had proximal flexor weakness but preserved distal grip strength. The right leg was short with pes cavus and hammertoes along with distal motor weakness and atrophy. The left leg showed no wasting and only mildly decreased strength in foot extensors.
Knee jerk reflexes were brisk bilaterally, but ankle jerk reflexes were absent. Sensory examination was normal. There was no cerebellar ataxia, but he had learning difficulties.

Nerve conduction studies showed markedly reduced asymmetric compound muscle action potential responses with dispersion and conduction slowing and significant conduction block at multiple sites fulfilling the AAEM 2003 criteria for definite multifocal motor neuropathy (figure $1, B$ and C). Sensory responses were within acceptable limits throughout. On EMG, neurogenic changes were observed in the wasted limbs but no evidence of recent denervation was found except in right deltoid on the most recent EMG requested after the patient complained of new onset shoulder weakness (tables e-1-e-4, links.lww.com/WNL/A481).

Imaging and laboratory studies including CSF were repeatedly unremarkable. Routine genetic diagnostic testing revealed normal CGH array and no pathogenic variants were detected on the next-generation panel for 56 genes associated with $\mathrm{CMT}^{2}$ Immunosuppressive therapy was tried without benefit.

We performed whole exome sequencing in the patient's genomic DNA. ${ }^{2}$ Variants were annotated and filtered against protein prediction, evolutionary conservation, and frequency in several databases and were validated by Sanger sequencing and segregation analysis in the patient and in his parents with special attention on variants in all known CMT-related genes. Immunoblotting was performed in fibroblasts by standard techniques. Enzyme function test was carried out using in vitro malachite green phosphatase assay as described. ${ }^{7}$ Label-free shotgun proteomic profiling utilizing patient-derived (4 biological replicates) and control fibroblasts ( 6 biological replicates) was performed (e-Methods, links.lww.com/WNL/A482).

\section{Data availability}

Whole exome sequencing data of the patient has been deposited in RD-CONNECT (rd-connect.eu/) and in the European Genome Phenome Archive (ega-archive.org/). The mass spectrometry data has been deposited to the ProteomeXchange Consortium 31 via the PRIDE partner repository with data identifier PXD008014 (proteomecentral.proteomexchange.org/cgi/GetDataset).

\section{Results}

The patient carried a novel de novo heterozygous c.269T $>$ C, p.(Phe90Ser) PTEN variant, which was absent in both parents 

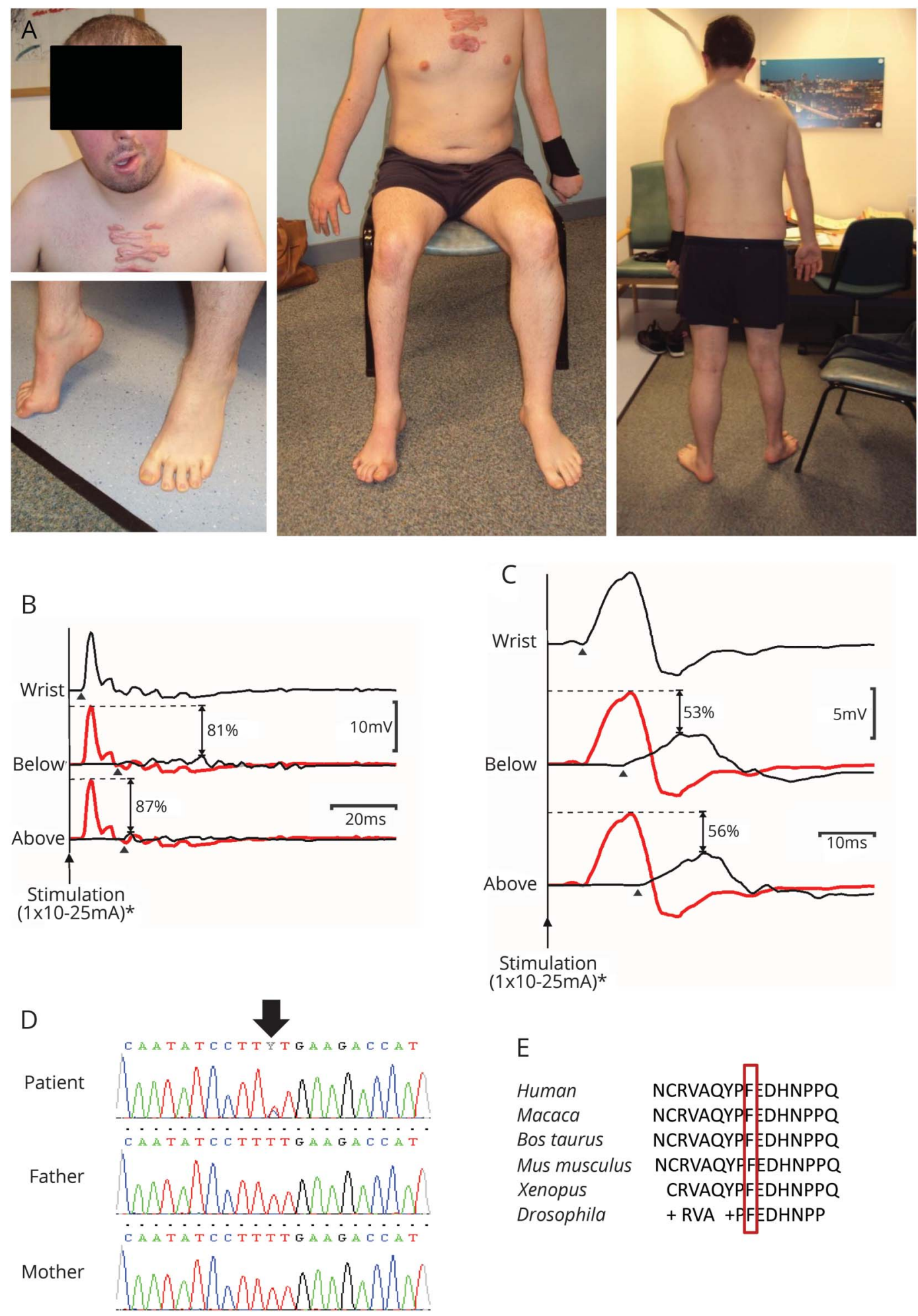

(A) Photographs of the patient show the asymmetric focal neuropathy. (B) Right abductor pollicis brevis (APB) compound muscle action potentials (CMAPs) obtained by stimulating the median nerve at the wrist (top), the antecubital fossa (middle), and above the elbow (bottom) demonstrate significant conduction block (with desynchronization) affecting the right median nerve within the forearm. In the lower traces, proximal responses (black lines) are superimposed on the distal CMAP (plotted in gray), thus facilitating visualization of the conduction block at each location (the percentage drop in CMAP amplitude at each site is also indicated). (C) Right abductor digiti minimi (ADM) CMAPs evoked by stimulating the ulnar nerve at the wrist (top) and below (middle) and above (bottom) the medial epicondyle demonstrate significant conduction block (with dispersion) affecting the right ulnar nerve within the forearm. Maximal distal CMAPs were obtained using stimulus currents of $\sim 10 \mathrm{~mA}$ and to confirm conduction block currents of $\sim 25 \mathrm{~mA}$ were used at proximal locations (pulse duration $0.5 \mathrm{~ms}$ ). Stimulus artefacts have been removed for clarity. Arrowheads mark CMAP onset. *Significantly higher stimulus currents (up to 100 mA) were used to confirm conduction block during clinical testing. (D) Electropherogram shows the de novo heterozygous c.269T>C, p.(Phe90Ser) PTEN mutation. (E) Evolutionary conservation of the mutation. 
(figure 1D). The mutation has not been reported in the ExAc database and it affects a highly conserved amino acid residue within the catalytic site of PTEN phosphatase domain and was predicted to be deleterious.

Immunoblotting revealed normal levels of PTEN in the patient's fibroblasts (figure 2, A and B). Enzyme function assay revealed no impaired p.(Phe90Ser) PTEN phosphatase activity on phosphatidylinositol 3,4,5-trisphosphate $\left(\mathrm{PIP}_{3}\right)$ as a substrate when compared the wild-type protein. However, there was a defect in catalytic activity against the secondary substrate of PTEN, phosphatidylinositol 3,4-trisphosphate $\left(\mathrm{PI}[3,4] \mathrm{P}_{2}\right)$, which was no longer apparent at high levels of $\mathrm{PI}(3,4) \mathrm{P}_{2}$ (figure $2, \mathrm{C}$ and $\mathrm{D}$ ).

Proteomic profiling in patient-derived fibroblasts revealed a total of 1,944 distinct proteins with altered abundance of 47 ( 9 proteins based on 2 or more peptides, 38 proteins on one unique peptide) with different subcellular localizations such as cytosol and cell membrane (figure 3 and figure e-1, links.lww. com/WNL/A480; e-Methods, links.lww.com/WNL/A482). LMCD1 and ITA3 are associated with cell migration and metastasis formation, ${ }^{8}$ while AK2, ILK, and SBP1 are involved in cellular antitumor response. ${ }^{9,10}$

\section{Discussion}

Germline PTEN mutations cause various human conditions with poorly understood genotype-phenotype correlations. ${ }^{4,11}$ PTEN shows limited genetic variation in the human population (1000 Genome Project found 54 single nucleotide variants with minor allele frequency $<0.001$ ) and the protein is highly conserved between species. Cytosolic PTEN consists of 403 amino acids divided into an $\mathrm{N}$-terminal phosphatase domain (7-185), a lipidbinding C2 domain (186-351), and a C-terminal tail (352-403), containing phosphorylation sites that regulate protein activity and stability. ${ }^{11}$ PTEN directly antagonizes Class I phosphoinositide 3-kinases ( $\mathrm{PI}[3] \mathrm{Ks})$ by converting $\mathrm{PIP}_{3}$ into phosphatidylinositol 4,5-bisphosphate $\left(\mathrm{PI}[4,5] \mathrm{P}_{2}\right)$. Diminished PI(3)K signaling exerts a brake on downstream effector pathways interfering with cellular processes such as cell growth, proliferation, and survival. ${ }^{5,11}$ The alternate PTEN substrate, $\mathrm{PI}(3,4) \mathrm{P}_{2}$, has many similar signaling functions to $\mathrm{PIP}_{3}$ and several tumors are caused by preferential loss of hydrolysis of $\mathrm{PI}(3,4) \mathrm{P}_{2}$ substrates $^{12}$ and depending on its subcellular location $\mathrm{PI}(3,4) \mathrm{P}_{2}$ is both a suppressor and enhancer of mTOR signaling. ${ }^{13}$

Loss of function germline mutations in PTEN are mainly associated with inherited tumors and severe PHTS, while retained or partially lost PTEN activity has been found in neurodevelopmental disorders. ${ }^{4}$ Besides its dual-specific phosphatase activity, phosphatase-independent mechanisms and protein-protein interactions have also been implicated in PTEN mutations. ${ }^{5}$

The p.(Phe90Ser) missense change identified in our patient is located within the WPD loop, which is equally near to the phosphatase active pocket and to the membrane-binding regulatory interface of the PTEN C2 domain. Most of the mutated WPD residues have been considered not to influence or to only partially affect PTEN catalytic activity. ${ }^{14}$ The somatic p.(Phe90Ser) PTEN mutation has been found in cancer (glioma, endometrium), and had normal phosphatase activity against $\mathrm{PIP}_{3}$ but a reduced ability to bind membrane phospholipids and recruit PTEN for $\mathrm{PIP}_{3}$ signalling. ${ }^{7}$ The germline c.269T $>\mathrm{C}$, p. (Phe90Ser) PTEN mutation in fibroblasts of our patient produced a stable protein with an unexpected deficit in activity against $\mathrm{PI}(3,4) \mathrm{P}_{2}$. This may be due to a novel allostery in the mutant or a change in the vulnerability of the protein to oxidation, which inhibits PTEN activity in these assays. In agreement with our data, a recent publication confirmed that PTEN is a $\mathrm{PI}(3,4) \mathrm{P}_{2}$ 3-phosphatase and together with INPP4B regulates $\mathrm{PI}(3,4) \mathrm{P}_{2}$ accumulation downstream of Class I PI3K, and $\mathrm{PI}(3,4) \mathrm{P}_{2}$ signaling may play a role in the tumor suppressor function of PTEN. ${ }^{15}$

The phenotype of this patient was unique and different from the neurologic presentations associated with PTEN defects reported previously. Extensive tumor screening did not detect any malignancies, but we considered his hypertrophic keloids as skin hamartomas. While fulfilling some of the clinical criteria of PHTS, including the presence of macrocephaly, skin lesions, and ASD, the predominant feature at presentation was an asymmetric, gradually progressive multifocal demyelinating motor neuropathy, with electrophysiologic evidence of definite partial conduction block fulfilling American Academy of Electrodiagnostic Medicine diagnostic criteria for multifocal motor neuropathy. ${ }^{16}$ A susceptibility to demyelination following mild trauma-similar to hereditary neuropathy with liability to pressure palsies (HNPP) - might explain the multifocal presentation in this disorder. In support of this theory, in a mouse model with targeted mutation of PTEN in Schwann cells, progressive peripheral neuropathy with focal hypermyelination, myelin outfoldings, and "tomacula" formation has been reported, showing striking similarities to the pathologic findings in HNPP. ${ }^{6}$ A similar clinical presentation with rapidly progressive asymmetric demyelinating neuropathy with conduction blocks and temporal dispersion affecting one limb, resembling an acquired inflammatory neuropathy, has been described in a patient with a FIG4 mutation, another rare cause of inherited demyelinating related to abnormal levels of PI 3phosphates. ${ }^{17}$ Furthermore, transheterozygous PTEN mutant Drosophila melanogaster have been found to have progressive motor function loss, with deficits in climbing and flight ability. ${ }^{18}$

Several genetic forms of CMT have been described with abnormal myelin histopathology and tomacula formation (CMT4B, CMT4H, CMT4F, CMT1B, and HNPP). ${ }^{1}$ The causative proteins contain phosphoinositide binding domains in CMT2B/RAB7, CMT4B1/MTMR2, CMT4B2/SBF2, CMT4J/FIG4, and CMT4H/FGD4, suggesting that abnormal $\mathrm{PI}(3) \mathrm{K}$ signaling is a common final pathway in demyelinating CMT (figure 2E). Changes in phosphoinositide metabolites can have many possible cellular effects interfering 
Figure 2 Functional effects of abnormal phosphatase and tensin homolog located on chromosome 10 (PTEN)

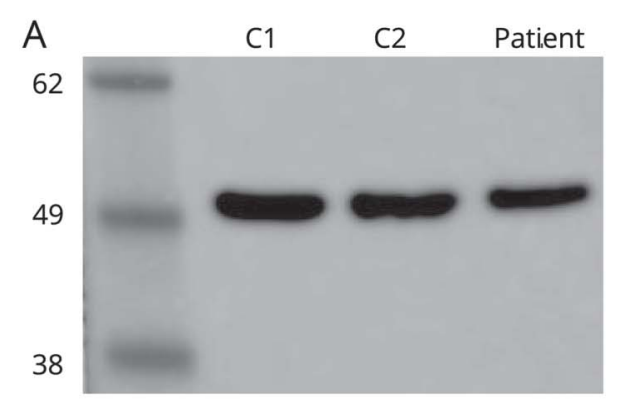

C. High-concentration diC8 substrates
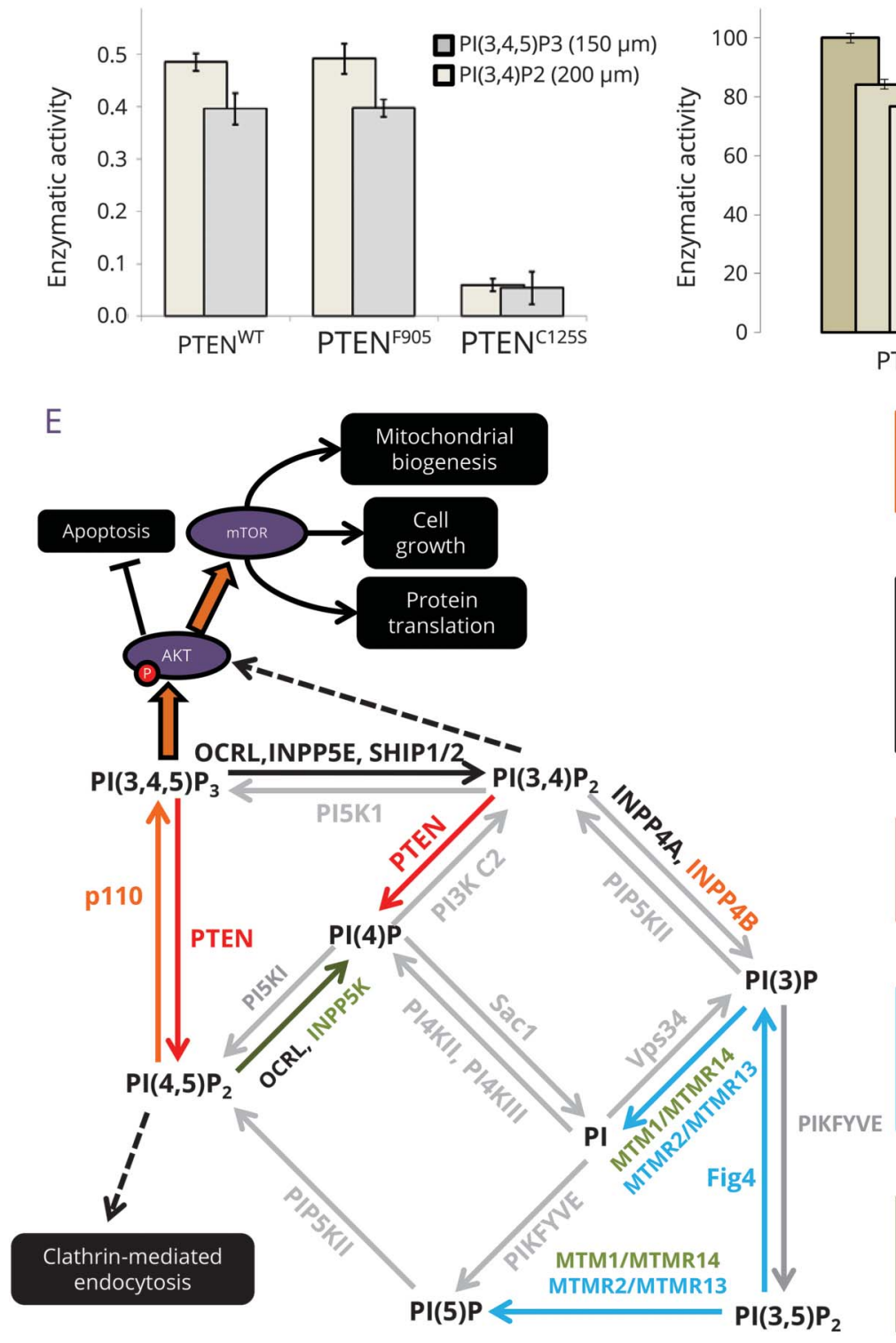

(A) Immunoblot (with marker size in $\mathrm{kDa}$ ) shows expression of PTEN in fibroblasts from 2 controls (C1, C2) and the patient. (B) Quantification of mean relative PTEN expression between controls ( $11, C 2$ ) and patient fibroblasts ( 3 replicates). (C) PTEN ${ }^{C 124 S}$ but not PTEN F90S shows reduced enzymatic activity against $150 \mu \mathrm{M} \mathrm{PI}(2,4,5) \mathrm{P}_{3}$ and $200 \mu \mathrm{M} \mathrm{PI}(3,4) \mathrm{P}_{2}$ compared to PTEN ${ }^{\mathrm{WT}}$. (D) PTEN ${ }^{\mathrm{F} 00}$ shows reduced activity against $<100 \mu \mathrm{M} P \mathrm{PI}(3,4) \mathrm{P}_{2}$ compared to PTEN ${ }^{\mathrm{T}}$. (E) The roles of PTEN within the phosphatidylinositol system, with human mutations causing neuropathy and cancer highlighted according to the key. 


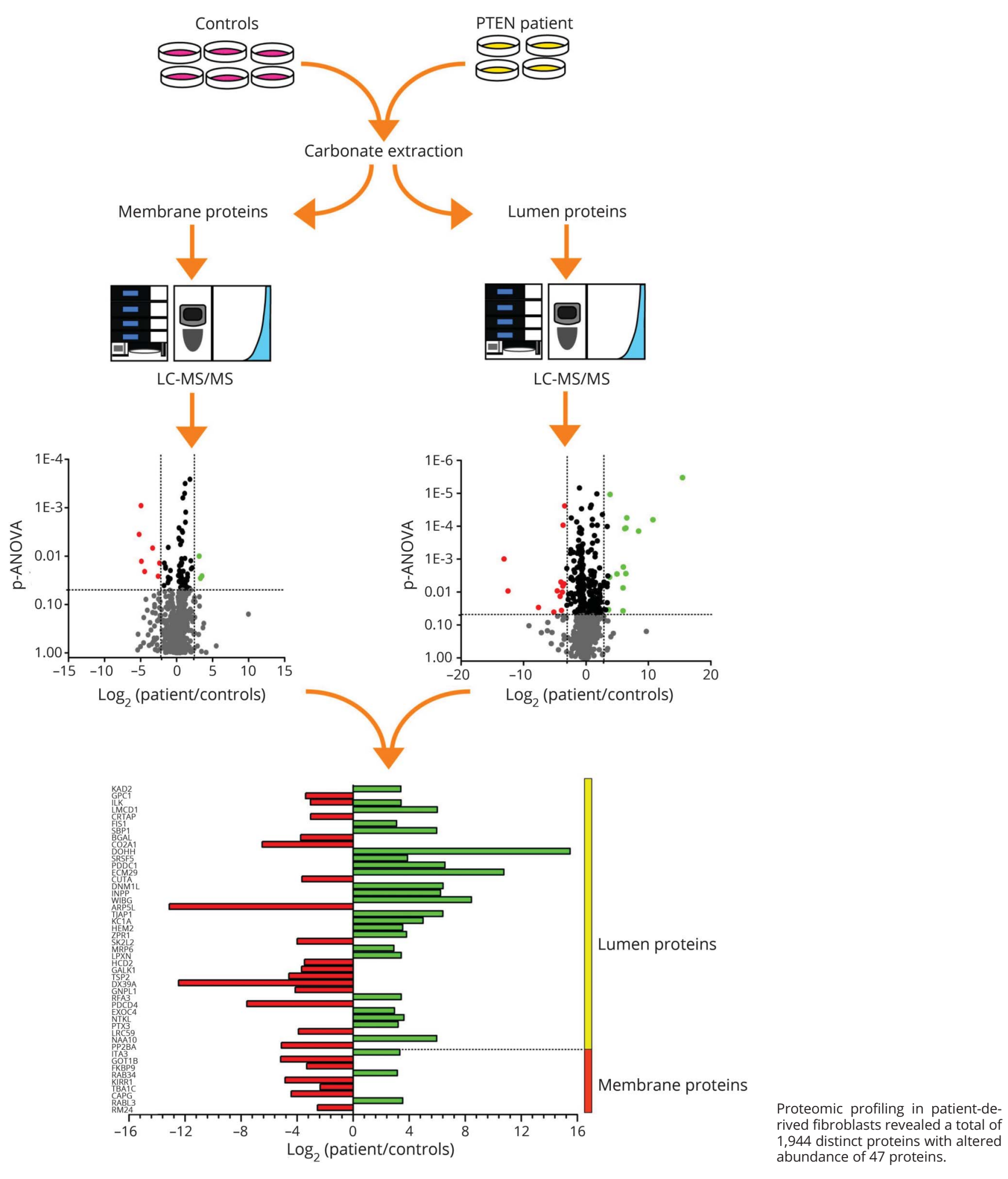

with normal myelin maintenance and the enzymes involved or their downstream targets are appealing therapeutic targets. Selective inhibition of $\mathrm{PI}(3) \mathrm{K}$ is a novel anticancer therapy approach already in clinical trials with favorable toxicity profile. ${ }^{19}$ Metabolomic studies of the same inhibitor show that plasma metabolites can be useful dose-dependent biomarkers of the response to therapy. ${ }^{20}$

Results of our proteomic profiling allowed the identification of several proteins supporting abnormal PTEN function: increased 
LMCD1 has been linked to tumor metastasis ${ }^{8}$ and ITA3 is involved in promoting cell invasion. ${ }^{21}$ In addition, we detected increased AK2, a negative regulator of tumor cell growth, ${ }^{9}$ indicating a compensatory effect, and may explain why extensive tumor screen did not detect malignant processes in our patient. In the same context, increased SBP1 may act as a tumor suppressor. ${ }^{10}$ Alteration of ITA3 and ILK suggests that integrin stimulation is a downstream effect of PTEN. ${ }^{22}$ We found several proteins, including GPC1, which are known to be regulated by PTEN activity and linked to peripheral myelination and axon guidance.

Our data revealed changes in expression of several proteins involved in tumorigenesis or tumor suppression, supporting a pathogenic role for the PTEN mutation, which may have caused the focal demyelinating motor neuropathy in association with a cutaneous hamartoma syndrome in our patient. We detected a concentration-dependent defect in catalytic activity of PTEN against its secondary substrate, $\mathrm{PI}(3,4) \mathrm{P}_{2}$, which may lead to multifocal demyelinating motor neuropathy in PTEN mutations and potentially in other CMTs with similar pathomechanisms.

\section{Author contributions}

B.B. participated in clinical examination, data collection, immunoblotting, and drafting of the manuscript. V.P., J.O., and A.R. performed the proteomic studies. M.J., J.S.M., and J.D. were responsible for the genetic and cell culture experiments. H.G. performed the bioinformatics analysis. J.A.L.M. and G.S.G. performed clinical examinations of the patient and reviewed the manuscript. M.R.B. and R.G.W. performed the electrophysiologic studies. A.R, L.E.S., and J.O. performed the enzymatic studies. P.F.C. and H.L. participated in the genetic analysis and in critical review of the manuscript. A.R and L.E.S. contributed to the drafting of the manuscript. R.H. is responsible for the experimental design, the clinical examination, and the drafting of the manuscript.

\section{Study funding}

R.H. is a Wellcome Trust Investigator (109915/Z/15/Z) who receives support from the Wellcome Centre for Mitochondrial Research (203105/Z/16/Z), Medical Research Council (UK) (MR/N025431/1), and the European Research Council (309548). H.L. receives funding from the European Union Seventh Framework Programme (FP7/2007-2013) under grant agreement no. 305444 (RD-Connect) and 305121 (Neuromics), from the Wellcome Trust Pathfinder Scheme (201064/Z/16/Z), and from the Newton Fund (UK/Turkey, MR/N027302/1). P.F.C. is a Wellcome Trust Senior Fellow in Clinical Science (101876/Z/13/Z) and a UK NIHR Senior Investigator, who receives support from the Medical Research Council Mitochondrial Biology Unit (MC_UP_1501/2), the Medical Research Council (UK) Centre for Translational Muscle Disease (G0601943), EU FP7 TIRCON, and the National Institute for Health Research (NIHR) Biomedical Research Centre based at Cambridge University Hospitals
NHS Foundation Trust and the University of Cambridge. A.R. received financial support from the Ministerium für Innovation, Wissenschaft und Forschung des Landes Nordrhein-Westfalen, the Senatsverwaltung für Wirtschaft, Technologie und Forschung des Landes Berlin and the Bundesministerium für Bildung und Forschung. L.E.S. was supported by Wellcome Trust (105616/Z/14/Z) and the Medical Research Council (MRC/ N010035/1).

\section{Disclosure}

The authors report no disclosures relevant to the manuscript. Go to Neurology.org/N for full disclosures.

Received October 29, 2017. Accepted in final form March 1, 2018.

\section{References}

1. Rossor AM, Evans MR, Reilly MM. A practical approach to the genetic neuropathies. Pract Neurol 2015;15:187-198.

2. Bansagi B, Griffin H, Whittaker RG, et al. Genetic heterogeneity of motor neuropathies. Neurology 2017;88:1226-1234.

3. Weis J, Claeys KG, Roos A, et al. Towards a functional pathology of hereditary neuropathies. Acta Neuropathol 2017;133:493-515.

4. Spinelli L, Black FM, Berg JN, Eickholt BJ, Leslie NR. Functionally distinct groups of inherited PTEN mutations in autism and tumour syndromes. J Med Genet 2015;52: 128-134.

5. Kreis P, Leondaritis G, Lieberam I, Eickholt BJ. Subcellular targeting and dynamic regulation of PTEN: implications for neuronal cells and neurological disorders. Front Mol Neurosci 2014;7:23.

6. Goebbels S, Oltrogge JH, Wolfer S, et al. Genetic disruption of Pten in a novel mouse model of tomaculous neuropathy. EMBO Mol Med 2012;4:486-499.

7. Nguyen HN, Yang JM Jr, Rahdar M, et al. A new class of cancer-associated PTEN mutations defined by membrane translocation defects. Oncogene 2015;34: 3737-3743.

8. Chang CY, Lin SC, Su WH, Ho CM, Jou YS. Somatic LMCD1 mutations promoted cell migration and tumor metastasis in hepatocellular carcinoma. Oncogene 2012;31: $2640-2652$.

9. Kim H, Lee HJ, Oh Y, et al. The DUSP26 phosphatase activator adenylate kinase 2 regulates FADD phosphorylation and cell growth. Nat Commun 2014; 5:3351.

10. Ansong E, Ying Q, Ekoue DN, et al. Evidence that selenium binding protein 1 is a tumor suppressor in prostate cancer. PLoS One 2015;10:e127295.

11. Leslie NR, Longy M. Inherited PTEN mutations and the prediction of phenotype. Semin Cel Dev Biol 2016;52:30-38.

12. Fedele CG, Ooms LM, Ho M, et al. Inositol polyphosphate 4-phosphatase II regulates PI3K/Akt signaling and is lost in human basal-like breast cancers. Proc Nat Acad Sci USA 2010;107:22231-22236.

13. Marat AL, Wallroth A, Lo WT, et al. mTORC1 activity repression by late endosomal phosphatidylinositol 3,4-bisphosphate. Science 2017;356:968-972.

14. Rodriguez-Escudero I, Oliver MD, Andres-Pons A, Molina M, Cid VJ, Pulido R. A comprehensive functional analysis of PTEN mutations: implications in tumor- and autism-related syndromes. Hum Mol Genet 2011;20:4132-4142.

15. Malek M, Kielkowska A, Chessa T, et al. PTEN regulates PI(3,4)P2 signaling downstream of class I PI3K. Mol Cel 2017;68:566-580.

16. Olney RK, Lewis RA, Putnam TD, Campellone JV. Consensus criteria for the diagnosis of multifocal motor neuropathy. Muscle Nerve 2003;27:117-121.

17. Cottenie E, Menezes MP, Rossor AM, et al. Rapidly progressive asymmetrical weakness in Charcot-Marie-Tooth disease type $4 \mathrm{~J}$ resembles chronic inflammatory demyelinating polyneuropathy. Neuromuscul Disord 2013;23:399-403.

18. Mensah LB, Davison C, Fan SJ, Morris JF, Goberdhan DC, Wilson C. Fine-tuning of PI3K/AKT signalling by the tumour suppressor PTEN is required for maintenance of flight muscle function and mitochondrial integrity in ageing adult Drosophila melanogaster. PLoS One 2015;10:e0143818.

19. Sarker D, Ang JE, Baird R, et al. First-in-human phase I study of pictilisib (GDC-0941), a potent pan-class I phosphatidylinositol-3-kinase (PI3K) inhibitor, in patients with advanced solid tumors. Clin Cancer Res 2015;21:77-86.

20. Ang JE, Pandher R, Ang JC, et al. Plasma metabolomic changes following PI3K inhibition as pharmacodynamic biomarkers: preclinical discovery to phase I trial evaluation. Mol Cancer Ther 2016;15:1412-1424.

21. Ren Y, Hao P, Law SK, Sze SK. Hypoxia-induced changes to integrin alpha 3 glycosylation facilitate invasion in epidermoid carcinoma cell line A431. Mol Cell Proteomics 2014;13:3126-3137.

22. Dey N, Crosswell HE, De P, et al. The protein phosphatase activity of PTEN regulates SRC family kinases and controls glioma migration. Cancer Res 2008;68: 1862-1871. 


\title{
Multifocal demyelinating motor neuropathy and hamartoma syndrome associated with a de novo PTEN mutation
}

\author{
Boglarka Bansagi, MD, PhD, Vietxuan Phan, PhD, and Mark R. Baker, PhD, FRCP, et al. \\ Cite as: Neurology ${ }^{\circledR}$ 2018;90:e1842-e1848. doi:10.1212/WNL.0000000000005566
}

\author{
Correspondence \\ Dr. Horvath \\ Rita.Horvath@ncl.ac.uk
}

\section{Study question}

Can PTEN mutations be associated with inherited demyelinating motor neuropathies?

\section{Summary answer}

Altered PTEN function may cause mulitfocal demyelinating motor neuropathy in association with a cutaneous hamartoma supported by changes in the expression of several proteins involved in tumorigenesis or tumor suppression.

\section{What is known and what this paper adds}

PTEN mutations have been associated with tumors, macrocephaly, autism, ataxia, tremor, and epilepsy in clinical patients. This report is the first to describe a multifocal demyelinating motor neuropathy and hamartoma syndrome in a patient with a de novo heterozygous PTEN mutation.

\section{Participants and setting}

A 29-year-old man exhibited delayed motor development, speech impediment, and facial asymmetry at 7 years of age. He subsequently developed focal weakness involving the right eye, face, and contralateral extremities, as well as multiple livid papules and keloid scars by the age of 20 . At 29 years of age, he presented with macrocephaly, autism spectrum disorder, and skin hamartomas, which are considered the clinical hallmarks for PTEN-related hamartoma tumor syndrome. Nerve conduction studies were consistent with the criteria for definite multifocal motor neuropathy.

\section{Primary outcomes}

The association of multifocal demyelinating motor neuropathy and hamartoma syndrome with a de novo heterozygous PTEN mutation.

\section{Design, size, and duration}

The patient's genome was sequenced with whole exome sequencing and variants were validated by Sanger sequencing and segregation analyses. Patient fibroblasts were harvested for labelfree shotgun proteomic profiling and biochemical analyses.

\section{Main results and the role of chance}

Sequencing revealed a novel de novo heterozygous c.269T $>$ C, p.(Phe90Ser) PTEN variant that was not present in either parent. The pathogenicity of the variant was further supported by a defect in fibroblast PTEN catalytic activity. Proteomic profiling revealed alterations in the expression of 47 proteins, including those associated

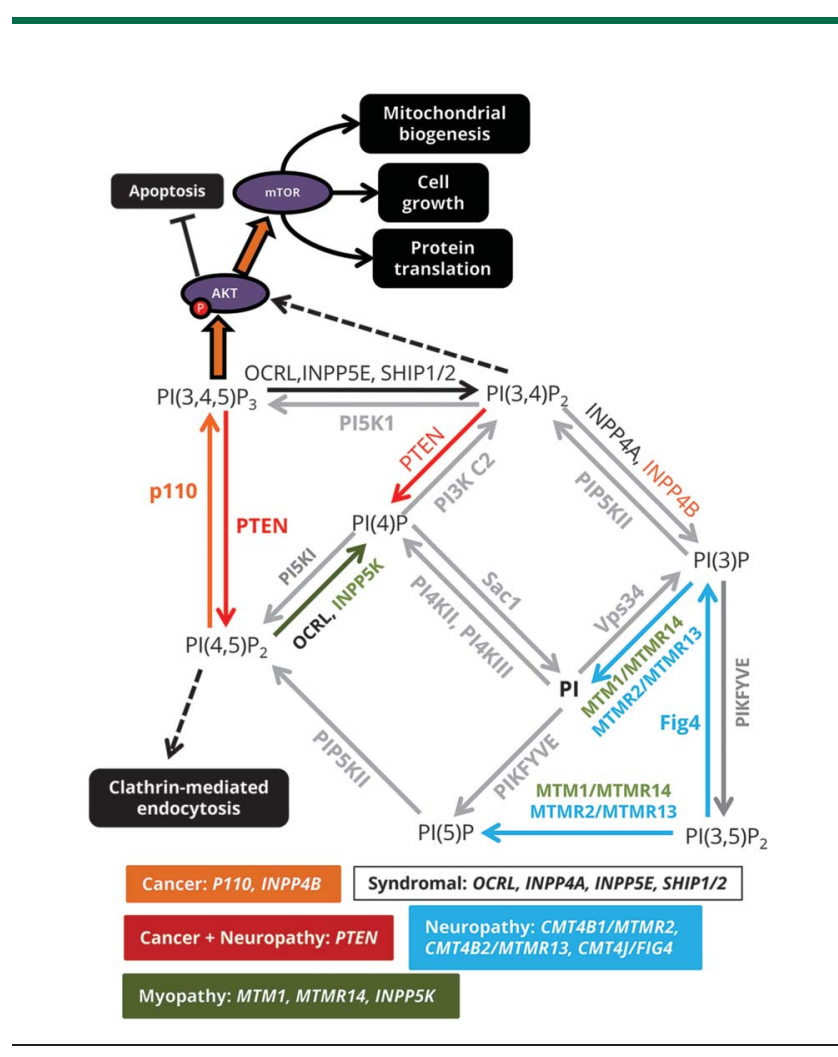

with cell migration and metastasis (LMCD1 and ITA3) and the cellular anti-tumor response (AK2, ILK, and SBP1).

\section{Bias, confounding, and other reasons for caution}

It is necessary to identify other patients carrying PTEN variants associated with multifocal motor neuropathy to confirm the association described in the present case.

\section{Generalizability to other populations}

The present findings can be generalized to patients with multifocal demyelinating neuropathy or other components of the phosphatidylinositol pathway.

\section{Study funding/potential competing interests}

The research was funded in part by the Wellcome Trust. Go to Neurology.org/N for full disclosures.

A draft of the short-form article was written by A. Symons, a writer with Editage, a division of Cactus Communications. The authors of the fulllength article and the journal editors edited and approved the final version. 


\section{Neurology}

\section{Multifocal demyelinating motor neuropathy and hamartoma syndrome associated with a de novo PTEN mutation}

Boglarka Bansagi, Vietxuan Phan, Mark R. Baker, et al.

Neurology 2018;90;e1842-e1848 Published Online before print May 2, 2018

DOI 10.1212/WNL.0000000000005566

\section{This information is current as of May 2, 2018}

\section{Updated Information \& Services}

\section{References}

Subspecialty Collections

Permissions \& Licensing

Reprints including high resolution figures, can be found at: http://n.neurology.org/content/90/21/e1842.full

This article cites 22 articles, 10 of which you can access for free at: http://n.neurology.org/content/90/21/e1842.full\#ref-list-1

This article, along with others on similar topics, appears in the following collection(s):

\section{All Genetics}

http://n.neurology.org/cgi/collection/all_genetics

Cranial neuropathy

http://n.neurology.org/cgi/collection/cranial_neuropathy

Peripheral neuropathy

http://n.neurology.org/cgi/collection/peripheral_neuropathy

Information about reproducing this article in parts (figures,tables) or in its entirety can be found online at:

http://www.neurology.org/about/about_the_journal\#permissions

Information about ordering reprints can be found online:

http://n.neurology.org/subscribers/advertise

Neurology ${ }^{\circledR}$ is the official journal of the American Academy of Neurology. Published continuously since 1951, it is now a weekly with 48 issues per year. Copyright Copyright ( 2018 The Author(s). Published by Wolters Kluwer Health, Inc. on behalf of the American Academy of Neurology.. All rights reserved. Print ISSN: 0028-3878. Online ISSN: 1526-632X.

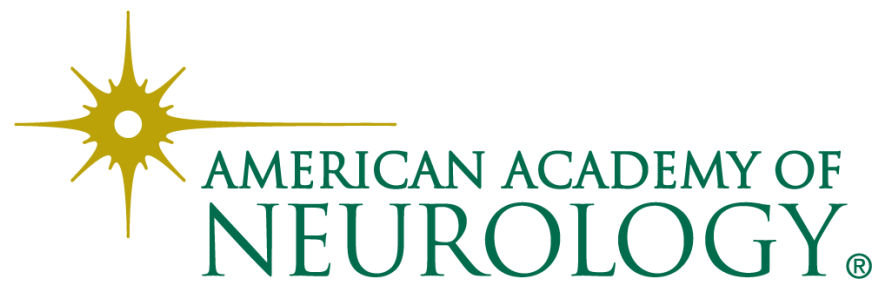

